\title{
MiR-I5b-5b Regulates the Proliferation of Prostate Cancer PC-3 Cells via Targeting LATS2
}

This article was published in the following Dove Press journal: Cancer Management and Research

\author{
Zhi-Jie Liu \\ Shi-Hui Liu \\ Jun-Ru Li \\ Xiao-Chuan Bie \\ Yang Zhou
}

Department of Urology, Hanting District People's Hospital of Weifang, Weifang, Shandong 261100, People's Republic of China
Correspondence: Zhi-Jie Liu

Department of Urology, Hanting District People's Hospital of Weifang, Weifang, Shandong 26I100, People's Republic of China

Email liuzhijie19898@I63.com
Purpose: In order to investigate the role of miR-15b-5b in the progression of prostate cancer.

Methods: We employed RT-qPCR assay to analyze the transcriptional level of miR-15b-5b in cell lines including PC-3, prostate cancer tissues as well as normal prostate tissues. The protein level of large tumor suppressor factor 2 (LATS2) was detected by Western blot in similar specimens. Bioinformatic analysis was used to predict the targets of miR-15b-5p, and dual-luciferase assay was performed to confirm the relationship of miR-15b-5p with LATS2. Cell proliferation assay and colony formation assay were used to assess the effects of miR$15 \mathrm{~b}-5 \mathrm{~b}$ on the proliferation of PC-3 cells. Multivariate analysis was performed to identify factors associated with overall survival using the Cox proportional hazards model.

Results: MiR-15b-5b was up-regulated in prostate cancer tissues as well as cell lines, and increased expression of miR-15b-5b was highly correlated with the poor prognosis of patients with prostate cancer. Ectopic expression of miR-15b-5b promoted the proliferation of PC-3 cells. Reciprocally, silence of miR-15b-5b elicited opposite effects on cell proliferation. Mechanistically, we identified LATS2 as the target of miR-15b-5b, which in turn limited LATS 2 expression in PC-3 cells. Furthermore, the stimulatory effects of miR-15b-5b on cell proliferation can be attenuated by overexpression of LATS2. Conversely, inhibition of LATS2 promoted the proliferation of PC-3 cells induced by miR-15b-5b. Our data thus demonstrate that dysregulation of miR-15b-5b exacerbates prostate cancer progression via suppression of LATS2.

Conclusion: The identification of the oncogenic role of miR-15b-5b in prostate cancer thus proposes that miR-15b-5p might be a new therapeutic target for the treatment of prostate cancer.

Keywords: prostate cancer, miR-15b-5b, LATS2, proliferation

\section{Introduction}

Prostate cancer is one of the most common malignancies in men, which ranks third among cancer-related deaths in men worldwide. ${ }^{1}$ At present, surgical resection is the main therapeutic measure, supplemented with radiotherapy, chemotherapy and endocrine therapy. ${ }^{2,3}$ Despite the advancement of prostate cancer treatment, there still are no definite measures were found. ${ }^{4}$ Therefore, it is urgent to figure out the pathogenesis of prostate cancer. Further investigation may provide a more effective or direct therapeutic measure for prostate cancer.

MicroRNAs (miRNAs) are small non-coding RNAs which serve as a significant role in the initiation and progression of multifarious types of tumor. ${ }^{5,6}$ Therefore, it is critical to investigate the mechanisms underlying the regulation of prostate cancer 
by miRNAs. MiR-15b is a member of the miR-15 family which is located on chromosome 3. MiR- $15 b-5 b$ is a mature miRNA spliced from the 5'- end of miR-15 precursor. $^{7}$ Up to now, a series of studies have shown that miR-15b-5b is involved in the regulation of a large number of tumor progression. ${ }^{8}$ On the one hand, miR-15b$5 \mathrm{~b}$ suppresses tumor progression by regulating signaling pathways. For example, miR-15b-5b promotes the apoptosis of colorectal cancer (CRC) cells via the NF- $\kappa B / X I A P$ axis. ${ }^{9}$ On the other hand, alteration of miR-15b-5b expression regulates tumor cell proliferation and migration. For example, overexpression of miR-15b-5b promotes migration and invasion of liver cancer cells. ${ }^{10}$ However, to the best of our knowledge, the mechanism of miR-15b-5b on prostate cancer is rarely reported.

Large tumor suppressor factor 2 (LATS2) is an important member of the LAST family, which plays a significant role in regulating the cell cycle, maintaining gene stability, inhibiting cell proliferation and inducing apoptosis. ${ }^{11}$ As a tumor suppressor gene, LATS2 exhibits low expression in a variety of tumors, and its high expression inhibits tumor progression. ${ }^{12}$ Research has shown that overexpression of LATS2 attenuates the growth-promoting effect of miR-302 or miR-327 on prostate cancer. ${ }^{12}$ However, whether miR-15b-5b affects the progression of prostate cancer by regulating LATS2 is not clear.

Here we found that miR-15b-5b was upregulated in prostate cancer tissues and PC-3 cells. Through targeting LATS2 mRNA, miR-15b-5b suppressed the expression of LATS2 and enhanced prostate cancer cell proliferation. In conclusion, our research thus identify the oncogenic role of miR-15b-5b in the development of prostate cancer and provides a theoretical basis for clinical treatment of prostate cancer.

\section{Materials and Methods}

\section{Human Tissue Specimens}

Prostate cancer specimens were obtained from the Hanting District People's Hospital of Weifang. The tumor tissues were collected from 30 patients with castration-resistant prostate cancer who underwent primary surgery between February 2018 and January 2019, and non-tumor tissues from 30 healthy donors were used for control experiments.

For all patients, the median age (range, years) was 45 (36-60), the median PSA at surgery was $8.9 \mathrm{ng} / \mathrm{mL}$, and miR-15b-5b showed high levels in the cancer tissues. All patients had histologically confirmed prostate adenocarcinoma (T2, 21 cases; T2-3 and T3, 9 cases). The participating patients would continue to track for 5 years, and the duration of their survival would be recorded after the operation. The study was approved by the Hanting District People's Hospital of Weifang. Written informed consent was obtained from all the participants.

\section{Cell Lines}

Human normal epithelial cell lines RWPE-1 and prostate cancer cell lines PC-3 were obtained from West China Hospital of Sichuan University. Keratinocyte serum-free medium supplemented with bovine pituitary extract and human recombinant EGF was used to culture RWPE-1 cell lines. PC-3 cells were cultured in DMEM medium, which supplemented with $10 \%$ FBS and $1 \%$ PenicillinStreptomycin solution. Cell lines were maintained in a $37^{\circ} \mathrm{C}$ incubator containing $5 \% \mathrm{CO}_{2}$.

\section{Cell Transfection}

Lipofectamine 2000 (Invitrogen, Thermo Fisher Scientific) mixed with miR-15b-5p mimics or miR-15b-5p inhibitor was performed to overexpress or suppress miR-15b-5b, corresponding mimics and inhibitors were purchased from Sangon Biotech (Shanghai, China). In detail, the mixture was maintained at room temperature for $20 \mathrm{~min}$ utes, then added into the cell culture medium, the cultured cells were harvested for related experiments following 72 hours transfection.

\section{LATS2 Overexpression and Silence}

Small interfering RNAs targeting LATS2 were synthesized by TSINGKE (Beijing, China), they were transfected into cells by using Lipofectamine 2000 to interfere with the expression of LATS2. For the overexpression, the full length of LATS2 was amplified from RWPE-1 cells cDNA and ligated into the pcDNA3.1 plasmid which was presented by Jilin University. The recombined plasmid was mixed with Lipofectamine 2000 in serum-free DMEM for 20 minutes and added into the culture medium. Following 72 hours transfection, the cultured cells were harvested for related experiments. The use of the cell lines was approved by the ethics committee of the Hanting District People's Hospital of Weifang.

\section{RNA Extraction and Real-Time RT-PCR}

TRIzol Reagent (Solarbia, life science) was used to extract the total RNA from prostate cancer tissues and cell lines. For miR-15b-5b mRNA level detection, RevertAid First Strand 
cDNA kit (Takara, Dalian, China) with stem-loop primer was used to reverse transcribe the total RNA. RT-qPCR was performed by using SYBR Premix Ex Taq kit (Takara, Dalian, China). U6 served as a control for miR-15b-5b normalization. $2^{-\Delta \Delta C t}$ methods were used to calculate the relative expression. Primers for q-RT-PCR were displayed as follow:

U6 Forward:5'-GCTTCGGCAGCACATATACTAAA AT-3';

U6 Reverse:5'-CGCTTCACGAATTTGCGTGTCAT-3';

GAPDH Forward: 5'-GGTGTGAACCATGAGAAG TATGA-3';

GAPDH Reverse: 5'-GAGTCCTTCCACGATACCAA AG-3';

$\beta$-actin Forward: 5'-GGACCTGACTGACTACCTC AT-3';

$\beta$-actin Forward: 5'-CGTAGCACAGCTTCTCCTTA AT-3';

miR-15b-5b Forward: 5'- ATCCAGTGCGTGTCG TG-3';

miR-15b-5b Reverse:5'- TGCTTAGCAGCACATCATG-3'.

\section{Luciferase Activity Assay}

WT or Mut 3'-UTR of LATS2 was amplified into psiCHECK2 reporter vector. Lipofectamine 2000 was used for co-transfection miR-15b-5b mimics and scrambling with WT 3'-UTR or Mut 3'-UTR of the LATS2 psiCHECK2 vector. Luciferase activity was detected and measured by the Dual-Luciferase Reporter Assay System (Promega) following cell harvesting and lysing following 72 hours transfection.

\section{Western Blot}

The protein was subjected to $10 \%$ SDS-page and transferred to a PVDF membrane (PVDF, Millipore, Billerica, MA). Then the PVDF membrane was placed in $4 \%$ milk for blocking. Primary antibodies against LAST2 (ab111054, Abcam, Cambridge, MA) and $\beta$ actin (ab8227, Abcam, Cambridge, MA) were used to incubate membranes overnight at $4^{\circ} \mathrm{C}$. On the next day, the PVDF membrane was washed and incubated with a secondary antibody for 1 hour at room temperature. In the end, Pierce ${ }^{\circledR}$ ECL (Pierce, Rockford, IL) Western blot Substrate's A and B solutions were mixed in a 1:1 ratio and evenly dropped onto the PVDF membrane. The signals were exposed to the image in the Tanon 5200 .

\section{Cell Proliferation Assay}

Cell Counting Kit-8 (Beyotime) was performed to analyze cell proliferation according to the instructions. In detail, cells were cultured in 96-well plate. $10 \mu \mathrm{L}$ CCK-8 solution was added into the culture medium in every well for 2 hours from 0-3 days. Then the medium mixed with CCK8 solution was transferred into a new 96-well plate. In the end, the fluorescent microplate reader was used to detect the absorbance at $450 \mathrm{~nm}$, which reflected cell proliferation ability.

\section{Colony Formation Assay}

Long-term cell proliferation was detected by colony formation assay. In detail, PC-3 cells transfecting with related plasmid or small interfering RNAs targeting LATS2 were inoculated into 12 -well plates at a dose of 50 cells per well. Dishes were taken out after cell clones in each well were over 25 . Numbers of cell clones were counted after staining with crystal violet.

\section{Statistical Analysis}

Comparisons between data groups were used paired $t$-test or one-way ANOVA by using GraphPad Prism ver. 6 (San Diego, CA). $P<0.05$ was considered a significant difference between groups. All resulting data were presented as mean \pm SEM of three independent experiments.

\section{Results}

\section{Dysregulation of MiR-15b-5b is Involved in the Progression of Prostate Cancer}

Dysregulation of MiR-15b-5b has been implicated in some tumors, such as hepatocellular carcinoma. ${ }^{13}$ To measure the status of miR-15b-5b in the development of prostate cancer, we employed RT-qPCR assay to detect the mRNA level of miR-15b-5b in prostate cancer tissues and cell lines. Using the reference genes including $\beta$-actin (ACTB), glyceraldehyde 3-phosphate dehydrogenase (GAPDH) or U6 for normalization of gene expression, the mRNA level of miR-15b-5b was upregulated in PC-3 cells as relative to normal epithelial cell line RWPE-1 (Figure 1A and B). Due to the similar trend of miR-15b-5b expression in PC-3 cells, we thus used $\mathrm{U} 6$ as the reference gene in the following experiments. As showing in Figure 1C, the levels of miR-15b$5 \mathrm{~b}$ were also up-regulated in prostate cancer tissues as relative to the normal tissues. Moreover, the 
A

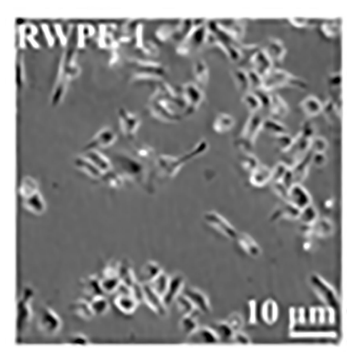

B
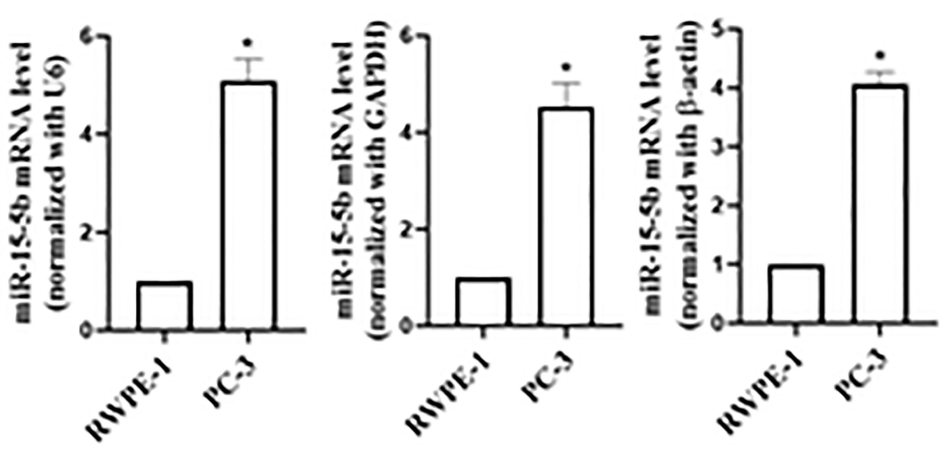

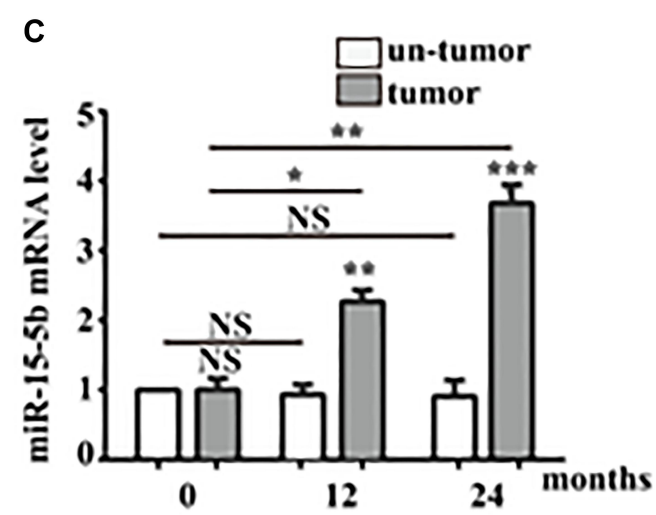

D

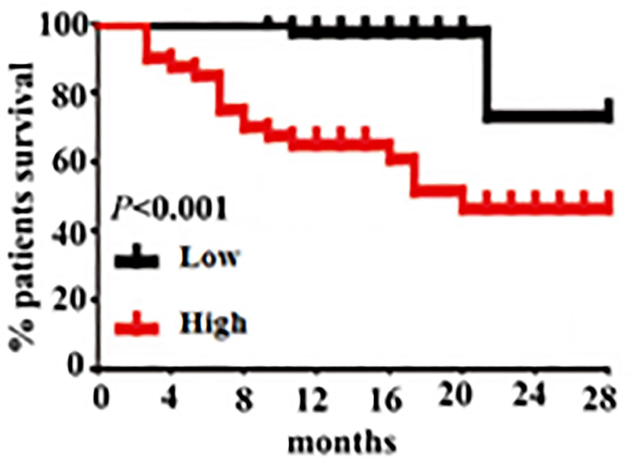

Figure I Transcriptional level of miR-15b-5b and its relationship with the survival rate of patients with prostate cancer. (A) REPE-I cells and PC-3 cells were observed under the microscope (scale bar, $10 \mu \mathrm{m}$ ). (B) RT-qPCR analysis of the mRNA level of miR-15b-5b in RWPE-I cells and PC-3 cells. (C) The mRNA level of miR-15b-5b in normal tissue specimen (un-tumor) compared with prostate cancer specimen (tumor) diagnosed in 0, 12, and 24 months. (D) Survival significantly decreased with the prolongation of prostate cancer. Comparisons were performed using the paired $t$-test. $*$ Indicates $p<0.05$, $* *$ Indicates $p<0.01$. $* * *$ Indicates $p<0.00$ I. NS indicates no significant difference. Error bars represent SEM. Data represent three independent experiments.

upregulation of miR-15b-5b was tightly correlated with tumor progression (Figure 1C). Accordingly, the multivariate analysis result showed that increased expression of miR-15b-5b was closely linked with poor outcome of patients with prostate cancer (Figure 1D). Our data thus suggest that miR-15b-5b is involved in the progression of prostate cancers.

\section{Ectopic Expression of MiR-I5b-5b Promotes Prostate Cancer Cell}

\section{Proliferation}

To investigate the biological function of miR-15b-5b in the development of prostate cancer, we analyzed the proliferative capacity of PC-3 cells in presence or absence of miR-15b-5b mimics (Figure 2A). Through the CCK8 detection assay, ectopic expression of miR-15b-5b mimics significantly promoted PC-3 cell proliferation as compared with miR-NCmimics did (Figure 2B). To further confirm this result, we used the colony formation assay and the results showed that the number of the colonies was significantly increased following miR-15b-5b overexpression compared with the control (Figure 2C and D). Our data thus demonstrate that upregulation of miR-15b-15 potentiates tumor growth of prostate cancers.

\section{MiR-I5b-5b Downregulation Limits Prostate Cancer Cell Proliferation}

To further investigate the role of miR-15b-5b in modulation of prostate cancer cell proliferation, we thus transfected the miR-15b-5b inhibitor into PC-3 cells to suppress endogenous miR-15b-5b (Figure $3 \mathrm{~A}$ ). In contrast to the enhanced cell proliferation of PC-3-expressing miR-15b-5b-mimics, the proliferation of PC-3 cells was decreased following the treatment of miR-15b-5b inhibitor (Figure 3B). In accordance with the CCK8 assay, the colony-forming capacity of PC-3 cells was also inhibited along with the inhibition of miR-15b-5b (Figure 3C and D). Above all, our results thus identify the oncogenic role of miR-15b-5b in the development of prostate cancers. 


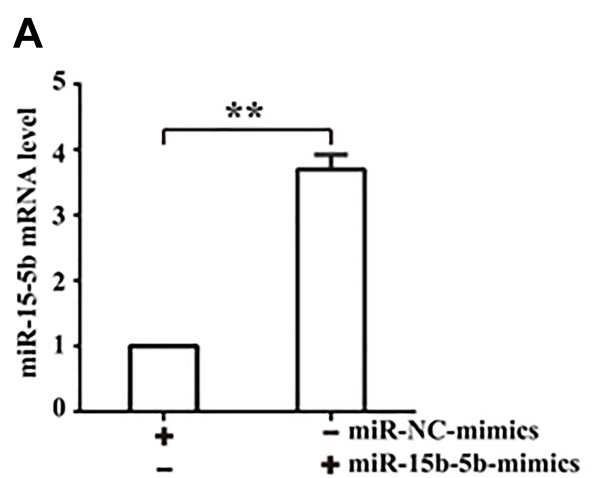

C

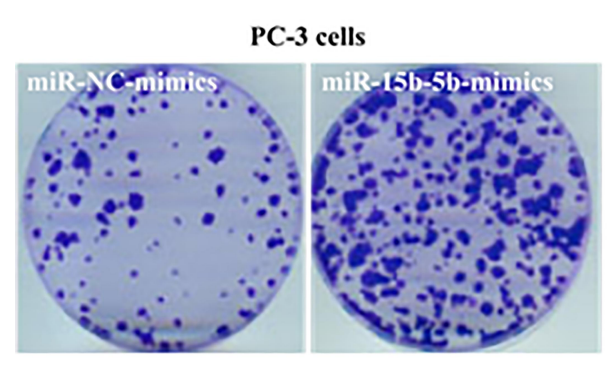

B

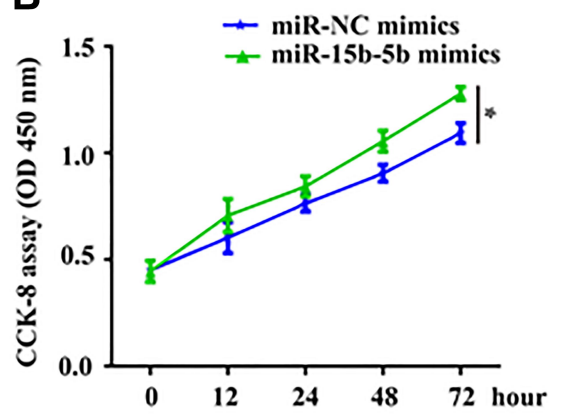

D

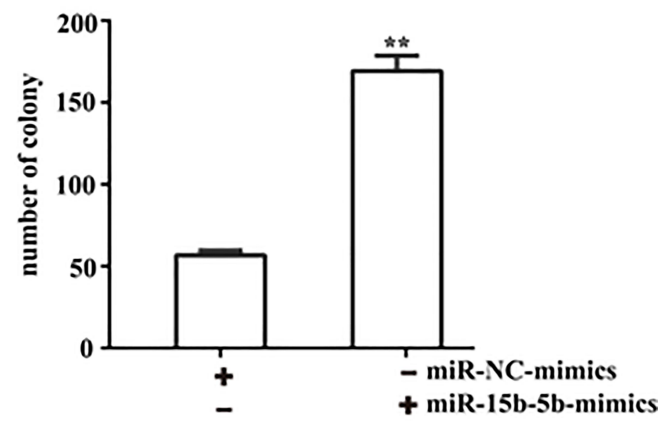

Figure 2 Overexpression of miR-I5b-5b promotes cell proliferation. (A) RT-qPCR assay was used to detect the transfection efficiency of miR-I5b-5b following miR-I5b-5b mimics transfecting. (B) Cell Counting Kit-8 was used to assess the proliferative rate of prostate cancer PC-3 cells at $0 \mathrm{~h}, 12 \mathrm{~h}, 24 \mathrm{~h}, 48 \mathrm{~h}$, and $72 \mathrm{~h}$ following miR-15b-5b overexpression. (C) Colony formation assay was employ to assess the growth of PC-3 cells, after miR-I5b-5b mimics transfecting, cells in each well were fixed and counted (Magnification: $\mid x$ ). (D) Counting the numbers of clones following the transfection with miR-I5b-5b-mimics or miR-NC-mimics. Comparisons were performed using the paired $t$-test. *Indicates $p<0.05$, **Indicates $p<0.0$ I. Error bars represent SEM. Data represent three independent experiments.

\section{MiR-I5b-5p Directly Binds to LATS2 mRNA and Restricts LATS2 Expression}

To investigate the mechanism by which miR-15b-5b promotes prostate cancer cell proliferation, we used miRanda to predict the potential target genes of miR-15b-5b. As shown in Figure $4 \mathrm{~A}$, miR-15b-5p directly targeted on the $3^{\prime}$-UTR in mRNA encoding large tumor suppressor factor 2 (LATS2). To further confirm this result, we used the Luciferase activity assay to detect the luciferase activity of related reporters in cell lines, including RWPE-1 and PC-3 cells. Following the cotransfection of miR-15b-5b mimics and psi-CHECK2 reporter vector, which contains WT or Mut 3'-UTR of LATS2, miR$15 \mathrm{~b}-5 \mathrm{~b}$ mimics significantly suppressed the luciferase activity of WT 3'-UTR of LATS2 in RWPE-1 cells, but not the Mut 3'UTR (Figure 4B). Consistently, as shown in Figure 4C, similar results were observed in PC-3 cells. According to the above results, our data suggest that miR-15b-5b directly targets and negatively regulates LATS2 expression.

\section{LATS2 Expression is Downregulated by MiR-I5b-5p in Prostate Cancer}

We next measured the expression of LATS2 in prostate cancers. As shown in Figure 5A, the protein level of
LATS2 was significantly down-regulated in prostate cancer tissue as relative to normal tissue. Similar result was also detected in PC-3 cells (Figure 5B). To further confirm the role of miR-15b-5b in regulation of LATS2 expression, we transfected the miR-15b-5b-mimics into PC-3 cells. As shown in Figure 5C, overexpression of miR-15b-5b significantly limited LATS2 expression by western blot assay. Reciprocally, inhibition of miR-15b-5b elicited opposite effects on LATS2 expression (Figure 5D). Our data thus uncover that LATS2 expression is down-regulated by miR$15 b-5 p$ in prostate cancer.

\section{LATS2 Overexpression Subverts MiR-I5b-5b-Induced Cell Proliferation}

To determine whether LATS2 is required for miR-15b-5binduced cell proliferation, we employed Lipofectamine 2000 to transfect recombinant plasmid (pcDNA3.1-LATS2) to rescue the down-regulation of LATS2 induced by miR-15b-5b in PC-3 cells. As shown in Figure 6A, overexpression of LATS2 rescued the downregulation of LATS2 induced by miR-15b-5b. As expected, through CCK-8 assay, overexpression of LATS2 significantly restricted miR-15b-5b-induced cell 
A

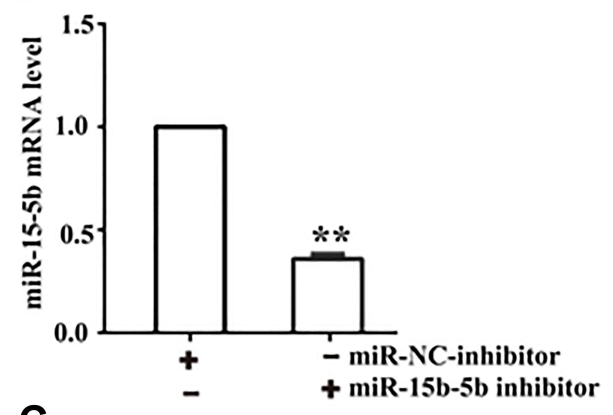

C

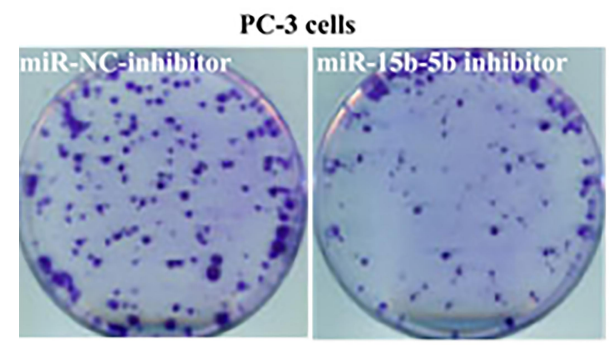

B

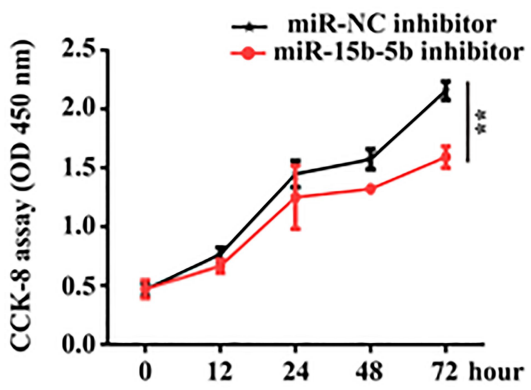

D

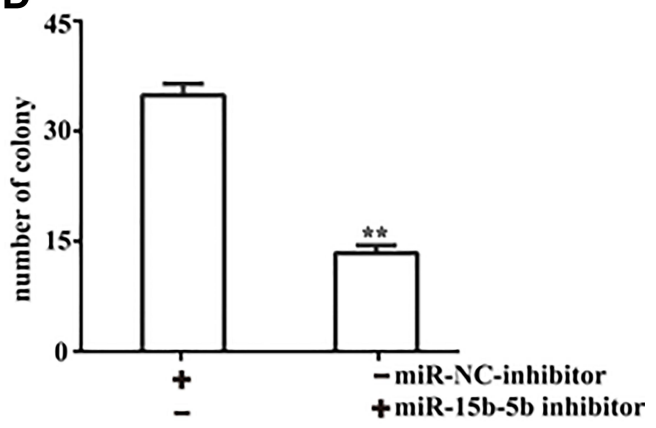

Figure 3 Downregulation of miR-I5b-5b inhibits the proliferation of prostate PC-3 cells. (A) qRT-PCR was performed to detect the expression of miR-I5b-5b following miR-I5b-5b suppressing. (B) Cell Counting Kit-8 (CCK-8) assay was used to assess the proliferation of PC-3 cells with or without miR-I5b-5b suppression. (C) The colonyforming capacity of prostate cancer PC-3 cells was detected by colony formation assay (Magnification:IX). (D) The number of the colonies was decreased following miR-I5b$5 b$ suppression compared with the control. Comparisons were performed using the paired $t$-test. $* *$ Indicates $p<0.0$ I. Error bars represent SEM. Data represent three independent experiments.

A

WT: 5'-CCCCCACCACUCGC- UGCUGCUU-LATS2 3'-UTR 111111

3'-ACAUUUGGUACUAC-ACGACGAU-5' miR-15b-5b I IIII

Mut: 5'- CCCCCACCACUCGC-UCGUGCUU-LATS2 3'-UTR
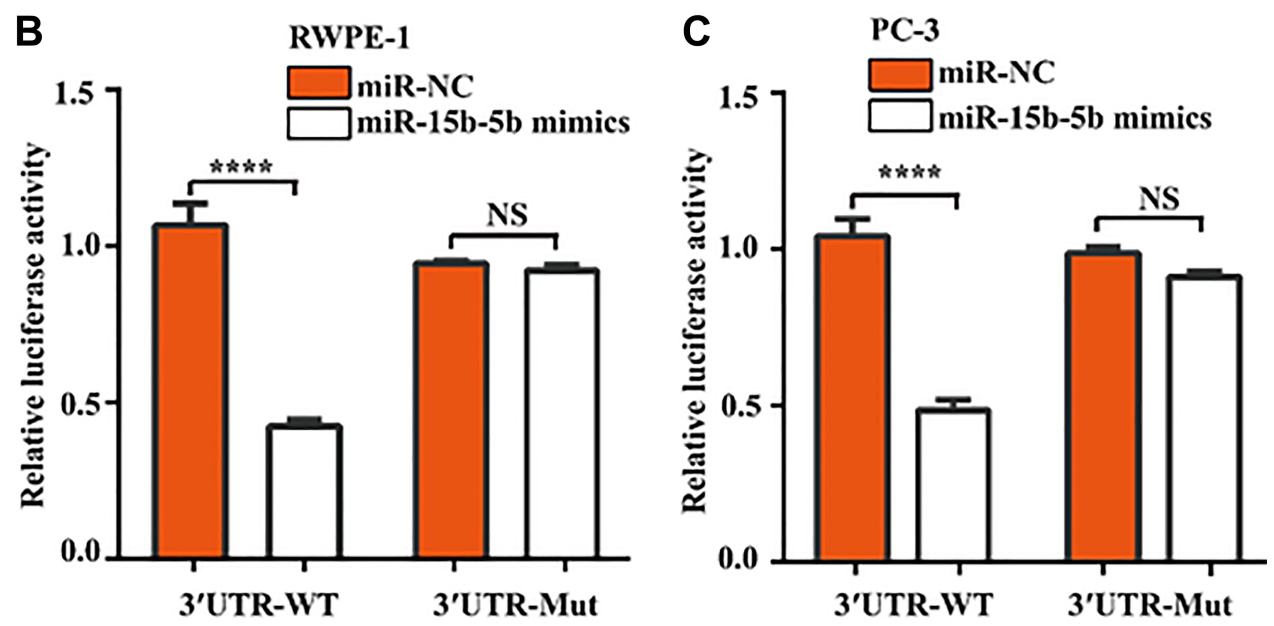

Figure 4 LATS2 acts as a target of miR-I5b-5b and negatively regulated by miR-I5b-5p. (A) miRanda was used to predict miR-I5b-5b targeted to WT or Mut 3'-UTR of LATS2. (B) After miR-15b-5b overexpression, luciferase activity assay was performed to detect the luciferase activity of reporters containing WT or Mut $3^{\prime}-$ UTR of LATS2 with miR-I5b-5p target sites in RWPE-I cells. (C) The same method was used to detect the luciferase activity in prostate cancer PC-3 cells. Comparisons were performed using the paired $t$-test. ${ }^{* * * *} p<0.000 \mathrm{I}$. NS indicates no significant difference. Error bars represent SEM. Data represent three independent experiments. 
A

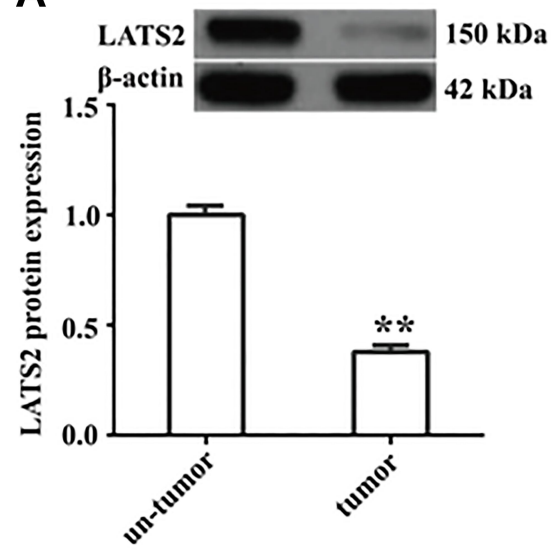

C

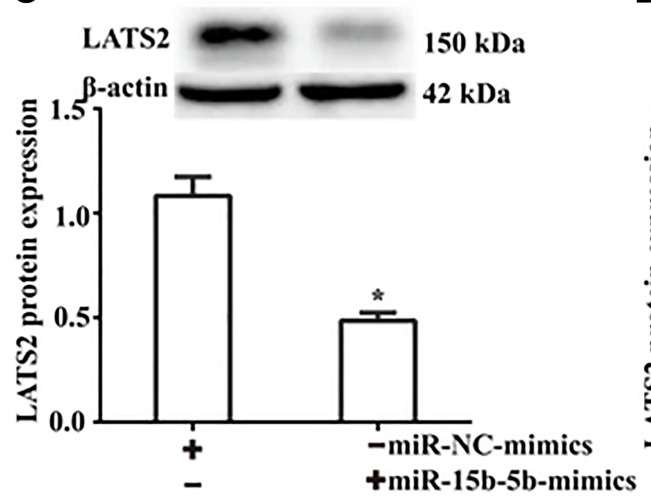

B

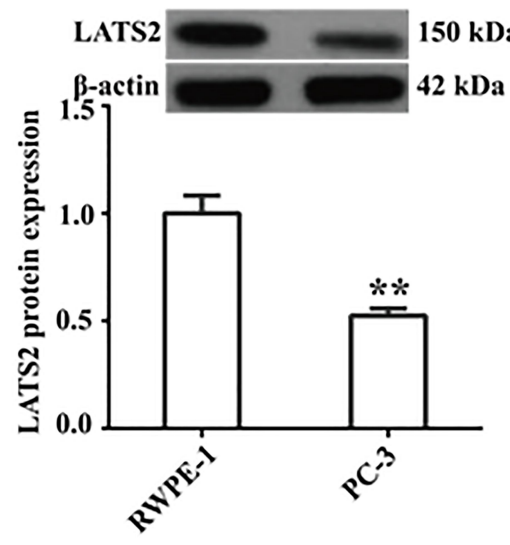

D

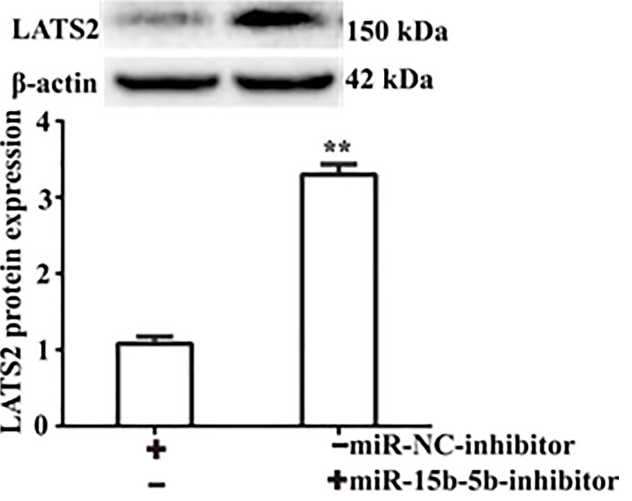

Figure 5 LATS2 expression is down-regulated by miR-15b-5p during prostate cancer development. (A) Western blot was performed to detect LATS2 protein expression in prostate cancer tissues. (B) LATS2 was down-regulated in prostate cancer PC-3 cells. (C) MiR-15b-5b restricted the expression of LATS2. (D) The expression of LATS2 was up-regulated following inhibition of miR-15b-15b. Comparisons were performed using the paired $t$-test. *Indicates $p<0.05$, $* *$ Indicates $p<0.01$. Error bars represent SEM. Data represent three independent experiments.

proliferation compared with the miR-NC-mimics did (Figure 6B). In addition, the colony formation assay results showed that the number of colonies induced by miR-15b-5b was significantly decreased following LATS2 overexpressing compared to the control (Figure $6 \mathrm{C}$ and D). These results thus demonstrate that the overexpression of LATS2 inhibits the stimulatory effects of miR-15b-5b on cell proliferation.

\section{Silence of LATS Strengthens MiR-I5b-5b- Mediated Cell Proliferation}

To further investigate the effect of LATS2 inhibition on the proliferation of PC-3 cells induced by miR-15b-5b, si-LATS2 was transfected into PC-3 cells, which overexpressed miR15b-5b. Western blot was performed to detect LATS2 expression (Figure 7A). We next employed the Cell Counting Kit-8 assay to assess the proliferation of PC-3 induced by miR-15b$5 \mathrm{~b}$ following LATS2 suppression. As shown in Figure 7B, PC3 cell proliferation induced by miR-15b-5b was significantly increased following LATS2 suppressing compared to the control did. Consistently, the number of colonies induced by miR$15 \mathrm{~b}-5 \mathrm{~b}$ was significantly increased following LATS2 suppression compared to the control (Figure 7C and D). Overall, our data demonstrate that miR-15b-5b promotes the proliferation of prostate cancer PC-3 cells via suppression of LATS2 expression.

\section{Discussion}

Prostate cancer is one of the most common malignancies in men, which seriously threat to human health. ${ }^{14}$ Further investigation may provide a more effective or direct therapeutic measure for prostate cancer. ${ }^{15}$ In the present study, our results indicated that miR-15b-5b was up-regulated in the prostate, and associated with poor prognosis of patients with prostate cancer. Besides, we found that miR-15b-5b promoted prostate cancer cell proliferation via suppression of LATS2 expression. Our data thus suggest that miR-15b-5b may serve as a potent therapeutic target for the treatment of prostate cancer. 
A

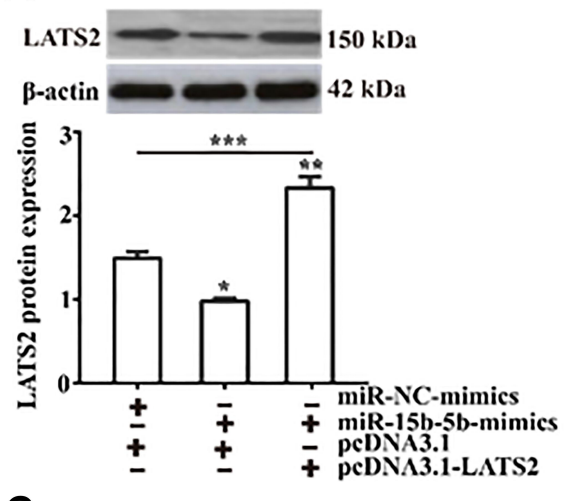

C

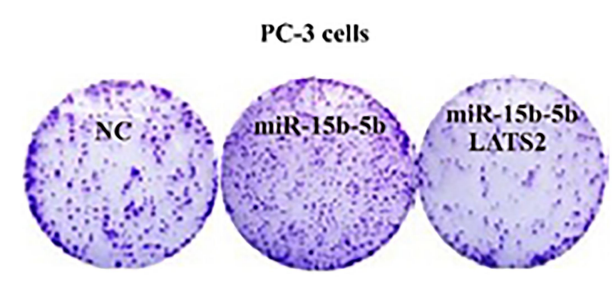

B

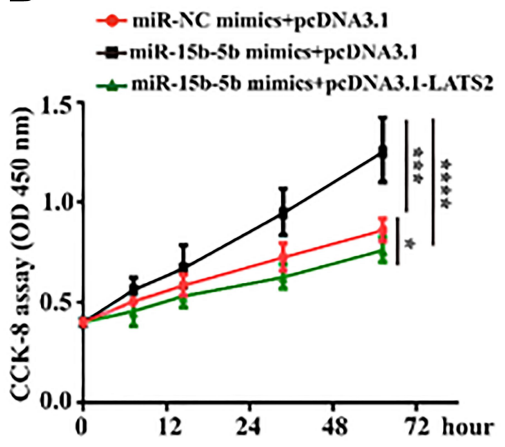

D

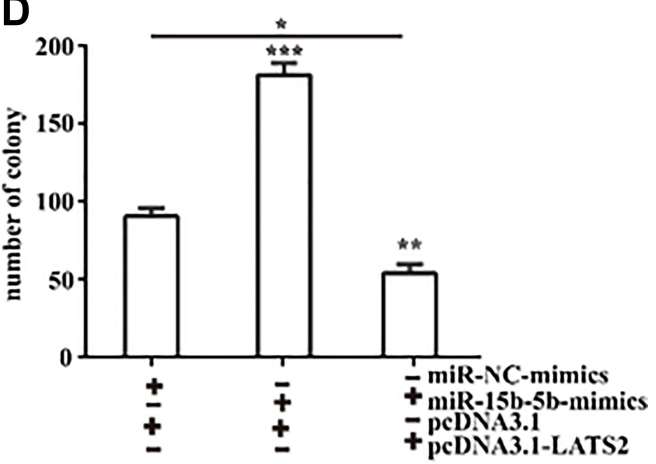

Figure 6 Overexpression of LATS2 attenuates miR-15b-5b-induced cells proliferation. (A) Western blot was performed to detect the expression of LATS2 protein in PC-3 cells with or without LATS2 overexpression following overexpression of miR-15b-5b. (B) The proliferation of PC-3 cells transfecting with the indicated vectors was analyzed by CCK8. (C) The colony-forming capacity of prostate cancer PC-3 cells with or without LATS2 overexpression after miR-I5b-5b overexpression was evaluated by colony formation assay (Magnification:Ix). (D) The proliferation of PC-3 cells induced by miR-I5b-5b was inhibited by LATS2. Comparisons were performed using the one-way ANOVA. *Indicates $p<0.05$, **Indicates $p<0.01$. $* * *$ Indicates $p<0.001$. ****Indicates $p<0.000$ I. Error bars represent SEM. Data represent three independent experiments.

MiR-15b-5b, which derive from miR-15b family. ${ }^{7}$ Increasing evidence has demonstrated that dysregulation of miR-15b-5b has been implicated in a series of tumor, and exacerbates cancer progression. ${ }^{16}$ Thus, understanding the regulation of miR-15b-5b on tumor progression is conducive to providing new ways for the treatment of tumors. Here our data uncover that miR-15b-5b is activated in prostate cancer and exacerbates cancer progression, which is consistent with previous researches that up-regulated miR-15b-5b accelerates tumor progression by promoting tumor cell proliferation. ${ }^{17,18}$ Moreover, inhibition of miR-15b-5b elicits the opposite effect on cancer cell proliferation. Further multivariate analysis showed that high expression of miR-15b-5b was associated with survival of prostate cancer patients. On the basis of these findings described above, miR-15b-5p may play a positive role in prostate cancer progression. We thus propose that miR-15b$5 p$ might be a new therapeutic target for prostate cancer.

MiRNAs play a significant role in the progression of a series of tumor via regulating tumor-associated gene expression. ${ }^{19,20}$ To further investigate the mechanisms underlying miR-15b-5b effect on prostate cancer, we aimed to explore the direct target gene of miR-15b-5b in prostate cancer. Bioinformatics analysis results indicated that that LATS2 is a target of miR-15b-5b. Besides, luciferase reporter assay showed that suppression of miR-15$5 \mathrm{~b}$ enhanced the luciferase activity of luciferase reporter containing LATS2 3'UTR WT, but not 3'UTR Mut. Furtherly, Western blot results indicated that LATS2 was negatively regulated by miR-15b-5p. These findings indicated that LATS2 is a direct target of miR-15b-5p and is negatively regulated by miR-15b-5p.

Served as a tumor suppressor, LATS2 tumor-suppressive effect has been proven in a large number of studies. ${ }^{15,21}$ In this study, we aimed to investigate the effect of LATS2 on the proliferation of PC-3 cells induced by miR-15b-5b. The results indicated that LATS2 inhibited PC-3 cell proliferation induced by miR-15b-5b, whereas LATS silence promoted proliferation. According to the results, we suggested that miR-15b-5b regulates the proliferation of prostate cancer PC-3 cells via targeting LATS2.

Above all, our study demonstrate that upregulation of miR-15b-5b promotes prostate cancer cell proliferation via 
A

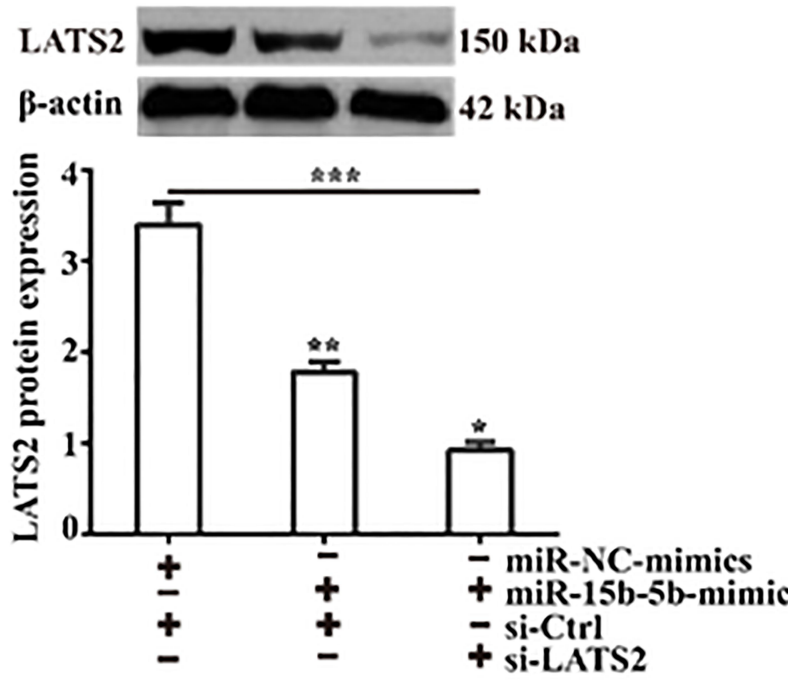

C

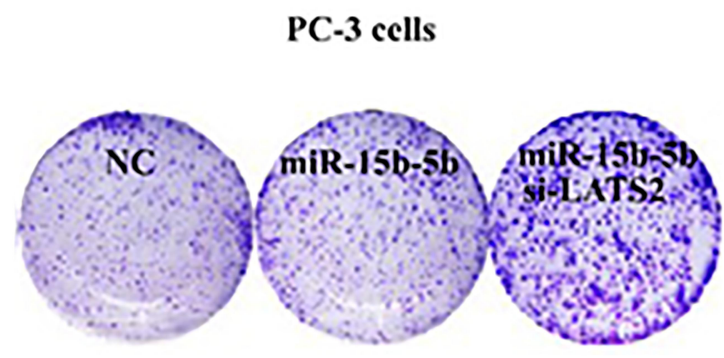

B

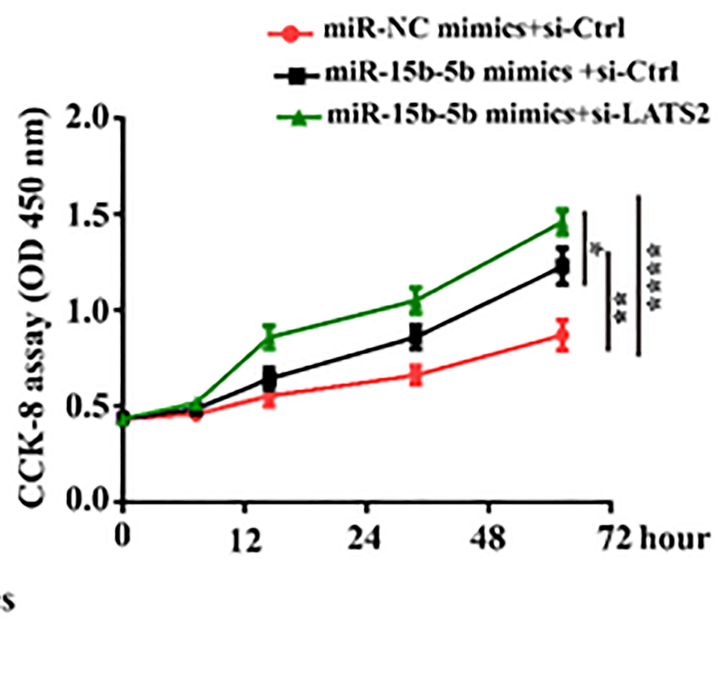

D

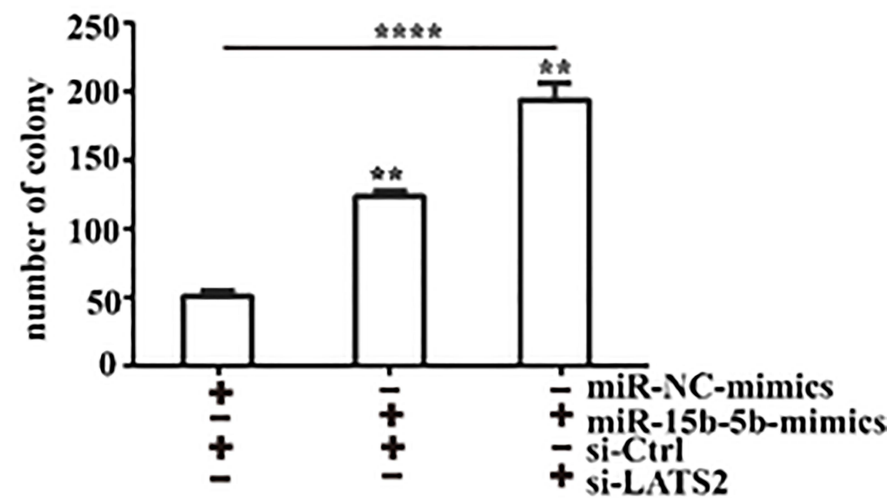

Figure 7 Silence of LATS promotes miR-15b-5b-induced cell proliferation. (A) The expression of LATS2 protein in PC-3 cells with or without LATS2 suppression following miR-I5b-5b overexpressing was detected by Western blot. (B) Cell Counting Kit-8 was used to evaluate the proliferation of PC-3 cells with or without LATS2 suppression following miR-15b-5b overexpressing. (C) Colony formation assay was performed to evaluate the colony-forming capacity of PC-3 cells with or without LATS2 suppression after miR-I5b-5b overexpression (Magnification:I $\times$ ); (D) LATS suppression promoted PC-3 cells proliferation induced by miR-I5b-5b. Comparisons were performed using the one-way ANOVA. *Indicates $p<0.05$, **Indicates $p<0.01$. ***Indicates $p<0.001$. *****Indicates $p<0.000$ I. Error bars represent SEM. Data represent three independent experiments.

suppression of LATS2 expression. We propose miR-15b$5 b$ may serve as a useful therapeutic target for prostate cancer and more studies are needed to confirm our findings in the future.

\section{Conclusion}

In summary, this study identifies the oncogenic role of miR-15b-5b in the development of prostate cancer via limitation of LATS2 expression. We thus propose miR$15 \mathrm{~b}-5 \mathrm{~b}$ may serve as a potent therapeutic target for the treatment of prostate cancer in the future.

\section{Funding}

The authors received no funding for this work.

\section{Disclosure}

The authors report no conflicts of interest in this work.

\section{References}

1. Aldrich T, Demark-Wahnefried W, Schildkraut JM, et al. The national cancer data base report on prostate cancer. Cancer. 1995;75(1):136-138. doi:10.1002/1097-0142(19950101)75:1<136:: AID-CNCR2820750126>3.0.CO;2-3

2. Taneja SS. Re: radical prostatectomy or watchful waiting on early prostate cancer. $J$ Urol. 2014;192(2):420-422. doi:10.1016/j.juro.20 14.05.075

3. Labrie F, Belanger A, Simard J, et al. Combination therapy for prostate cancer: endocrine and biologic basis of its choice as new standard first-line therapy. Cancer. 2015;71(S3):1059-1067.

4. Zagars GK, Eschenbach ACV, Ayala AG. Prognostic factors in prostate cancer: analysis of 874 patients treated with radiation therapy. Cancer. 1993;72(5):1709-1725. doi:10.1002/1097-0142(19930901) 72:5<1709::AID-CNCR2820720535>3.0.CO;2-O 
5. Fang YX, Gao W-Q. Roles of microRNAs during prostatic tumorigenesis and tumor progression. Oncogene. 2014;33(2):135-147. doi:10.1038/onc.2013.54

6. Kohlhapp FJ, Mitra AK, Lengyel E, et al. MicroRNAs as mediators and communicators between cancer cells and the tumor microenvironment. Oncogene. 2015;34(48):5857-5868. doi:10.1038/onc.2015.89

7. Finnerty JR, Wang W-X, Hébert SS, et al. The miR-15/107 group of microRNA genes: evolutionary biology, cellular functions, and roles in human diseases. J Mol Biol. 2010;402(3):491-509. doi:10.1016/j. jmb.2010.07.05

8. Li J, Chen Y, Guo X, et al. Inhibition of miR-15b decreases cell migration and metastasis in colorectal cancer. Tumour Biol. 2016;37 (7):8765-8773. doi:10.1007/s13277-015-4396-9

9. Zhao C, Zhao Q, Zhang C, et al. miR-15b-5p resensitizes colon cancer cells to 5 -fluorouracil by promoting apoptosis via the NF- $\mathrm{B} / \mathrm{XIAP}$ axis. Sci Rep. 2017;7(1):4194. doi:10.1038/s41598-017-04172-Z

10. Zhang Y, Huang F, Wang J, et al. MiR-15b mediates liver cancer cells proliferation through targeting BCL-2. Int J Clin Exp Pathol. 2015;8 (12): 15677.

11. Dong C, Wei K-J, Zhang W-B, et al. LATS2 induced by TNF-alpha and inhibited cell proliferation and invasion by phosphorylating YAP in oral squamous cell carcinoma. J Oral Pathol Med. 2015;44 (6):475-481. doi:10.1111/jop.12317

12. Shi Y, Geng D, Zhang Y, et al. LATS2 inhibits malignant behaviors of glioma cells via inactivating YAP. J Mol Neurosci. 2019;68 (1):38-48. doi:10.1007/s12031-019-1262-Z

13. Yang Y, Hou N, Wang $X$, et al. miR-15b-5p induces endoplasmic reticulum stress and apoptosis in human hepatocellular carcinoma, both in vitro and in vivo by suppressing Rab1A. Oncotarget. 2015;6 (18):16227-16238. doi:10.18632/oncotarget.3970
14. Dall'Era MA, Davies BJ, Eggener S. Active surveillance for prostate cancer. Eur Urol. 2012;62(6):976-983. doi:10.1016/j.eururo.2012. 05.072

15. Zhao C, Wang G, Zhu Y, et al. Aberrant regulation of miR-15b in human malignant tumors and its effects on the hallmarks of cancer. Tumour Biol. 2016;37(1):177-183. doi:10.1007/s13277-015-4269-2

16. Kanwal R, Plaga AR, Liu X, et al. MicroRNAs in prostate cancer: functional role as biomarkers. Cancer Lett. 2017;407:9-20. doi:10.1016/j.canlet.2017.08.011

17. $\mathrm{Lu} \mathrm{L,} \mathrm{Li} \mathrm{Y,} \mathrm{Wen} \mathrm{H}$, et al. Overexpression of miR-15b promotes resistance to sunitinib in renal cell carcinoma. J Cancer. 2019;10 (15):3389-3396. doi:10.7150/jca.31676

18. Fu Y, Wang C, Zhang D, et al. miR-15b-5p ameliorated high glucose-induced podocyte injury through repressing apoptosis, oxidative stress, and inflammatory responses by targeting sema3A. J Cell Physiol. 2019;234(11):20869-20878. doi:10.1002/jcp.28691

19. Gao G, Xiu D, Yang B, et al. miR-129-5p inhibits prostate cancer proliferation via targeting ETV1. Onco Targets Ther. 2019;12:3531-3544. doi:10.2147/OTT.S183435

20. Wang H, Ou J, Jian Z, et al. miR-186 modulates hepatocellular carcinoma cell proliferation and mobility via targeting MCRS1-mediated Wnt/beta-catenin signaling. $J$ Cell Physiol. 2019;234(12):23135-23145. doi:10.1002/jcp.28878

21. Zhang L, Li S, Wang R, et al. Anti-tumor effect of LATS2 on liver cancer death: role of DRP1-mediated mitochondrial division and the Wnt/beta-catenin pathway. Biomed Pharmacother. 2019;114:108825. doi:10.1016/j.biopha.2019.108825

\section{Publish your work in this journal}

Cancer Management and Research is an international, peer-reviewed open access journal focusing on cancer research and the optimal use of preventative and integrated treatment interventions to achieve improved outcomes, enhanced survival and quality of life for the cancer patient.
The manuscript management system is completely online and includes a very quick and fair peer-review system, which is all easy to use. Visit http://www.dovepress.com/testimonials.php to read real quotes from published authors. 